\title{
Proyecto "Aprendamos Jugando". Importancia del trabajo interdisciplinario en el paciente nacido prematuro y su grupo familiar en un área de alto riesgo socioambiental The "Learning Through Play" project. Importance of interdisciplinary work among children born prematurely and their family groups in an area of high socio-environmental risk
}

Dra. Flor L. Tobar ${ }^{a}$ L Lic. Manuel J. Lencina ${ }^{b}$

\section{RESUMEN}

Diversos factores de riesgo socioambiental determinan que un gran número de niños prematuros sin patología neurológica presenten bajos puntajes en su desarrollo, particularmente, en las áreas cognitiva y socioemocional, lo que evidencia un potencial riesgo futuro. El fortalecimiento de los vínculos familiares o con los cuidadores es fundamental para su adecuado progreso.

Este trabajo relata la experiencia de un equipo interdisciplinario del Hospital Eva Perón de Tucumán, que diseñó el programa de promoción del desarrollo infantil "Aprendamos Jugando", dirigido a los padres de los prematuros del Consultorio de Seguimiento y Servicio Social, con colaboración del Servicio de Nutrición. Palabras clave: prematuro, riesgo ambiental, intervención, vinculo, cognición.

http: / / dx.doi.org/10.5546/ aap.2017.588

Texto completo en inglés:

http: / / dx.doi.org/10.5546/ aap.2017.eng.588

Salí, provincia de Tucumán, Argentina.

b. División Servicio Social, Unidad de Gestión Paciente, Hospital Eva Perón de Tucumán, Banda del Río Salí, provincia de Tucumán, Argentina.

Correspondencia: Dra. Flor L. Tobar: florltobar@gmail.com

Financiamiento:

Ninguno.

Conflicto de intereses:

Ninguno que declarar.

Recibido: 30-9-2016

Aceptado: 10-5-2017

\section{INTRODUCCIÓN}

Indicadores como pobreza, enfermedades familiares, problemas socioemocionales, violencia, maltrato y abuso familiar hacen que un gran número de niños nacidos prematuros sin evidencia de trastornos neurológicos no sigan el ritmo de desarrollo esperado para la edad, lo que aumenta su vulnerabilidad y afecta su calidad de vida y posibilidades futuras. ${ }^{1}$ Estas alteraciones, habitualmente, moderadas, se ven reflejadas en un menor rendimiento académico y, sobre todo, adaptación social, con respecto a sus pares. ${ }^{2,3}$ En general, aquellos que presentan un trastorno motor evidente acceden a centros de intervención. Sin embargo, el resto, como en este caso, no cuentan con un sistema público que los pueda contener y enseñar. Así, se ven afectados en esos dos primeros años del período crítico del desarrollo por su alta vulnerabilidad e importancia del anclaje de las bases de las estructuras del desarrollo para una futura inserción integral adecuada en la sociedad, con un riesgo potencial evidente. ${ }^{4-6}$

En los niños de este grupo etario nacidos prematuros en seguimiento en Consultorio y Servicio Social del Hospital Eva Perón de Tucumán, se observaron estas dificultades. Utilizando el test de aprendizaje y desarrollo infantil (TADI), ${ }^{7}$ reflejaban puntajes totales 
normales, con parciales más bajos en las áreas socioemocionalidad y cognición.

Buscando el causal común, se observó que sus familiares no tuvieron experiencias de juego o socialización adecuadas que pudieran transmitir a sus hijos en forma positiva. Ante la necesidad de una intervención lo más temprana y oportuna posible en este período crítico, ${ }^{8-10}$ se planteó la creación de una herramienta en la que la terapéutica se implementara a través de la experiencia con los padres y sus familias: talleres vivenciales de promoción del desarrollo infantil "Aprendamos Jugando". Así, a través del juego, se formulaban sus realidades de vida y se trabajaban diferentes valores, como, por ejemplo, el ser persona, que se detallan más adelante. Estos valores elegidos como principios nos permiten orientar nuestro comportamiento en función de realizarnos como personas de bien.

Se relata la experiencia vivida, su impacto en el medio familiar y el mejoramiento del puntaje en las áreas más afectadas, y se destaca que fue el único hospital provincial que realizaba estos talleres sola y enteramente elaborados y conducidos por la interacción del Consultorio de Seguimiento, Servicio Social y la colaboración del Servicio de Nutrición.

\section{OBJETIVO DEL PROGRAMA}

Posibilitar a los padres encontrar sus propias herramientas a partir de la experiencia personal de juego y, a través de este, incentivar y favorecer la interacción con sus hijos, sobre la base del fortalecimiento del vínculo y del trabajo de valores (modelo multimodular). ${ }^{11}$

\section{UBICACIÓN GEOGRÁFICA, POBLACIÓN Y SITUACIÓN DE RIESGO SOCIAL}

El Hospital Eva Perón está ubicado en Banda del Río Salí, provincia de Tucumán, lindante con la capital. Referente del Área Programática Este, está compuesta por una vasta población de riesgo socioambiental, que predispone y responde a los indicadores de riesgo para presentar partos prematuros, entre ellos, los socioambientales, ${ }^{12-14}$ que corresponden, en su gran mayoría, a Alderetes y La Banda. Además de la excelencia científica en la prestación, entre otras cosas, se caracteriza por centrar la atención en función de las necesidades de la comunidad, mediante la escucha activa y respetando sus derechos y culturas. Al Programa de Seguimiento ingresan bebés nacidos prematuros que pesaron $1500 \mathrm{~g}$ o más al nacer.
Para el Servicio Social de Seguimiento Neonatal, se consideran con riesgo social aquellos pacientes cuyos padres presentan lo siguiente: ${ }^{15}$

- Nivel socioeconómico medido con indicadores de pobreza.

- Retraso mental o enfermedad psiquiátrica.

- Abuso de sustancias tóxicas.

- Antecedentes de maltrato o abandono de otros hijos.

- Menores de edad que no realizaron control prenatal.

- Otros menores discapacitados en el hogar.

- Problemas de alfabetización.

De los pacientes del Consultorio de Seguimiento atendidos en forma conjunta con Servicio Social, se seleccionaron 32 (10 mujeres y 22 varones) de entre 32 y 36 semanas de edad gestacional y mayores de $1500 \mathrm{~g}$ al nacer y que tenían, como mínimo, 2 factores de riesgo socioambiental mencionados. El rango etario fue entre 8 y 24 meses de edad corregida, de manera que los padres pudieran interactuar más activamente con ellos. Por la edad, dos comenzaron a partir del segundo taller. La mayoría no presentaban trastorno del desarrollo evidente, aunque, en consultorio, no se mostraban colaborativos ni sociables.

Coincidentemente, al evaluarlos con el TADI, 22 presentaban puntaje total (T) normal y 9, normal con rezago (riesgo en el área socioemocionalidad).

Sin embargo, en general, los puntajes en las áreas socioemocionalidad y cognición eran más bajos con respecto a las áreas motricidad y lenguaje. En esta última, 3 tenían puntajes de riesgo. Uno presentaba diagnóstico neurológico de "retraso" y antecedente de meningitis: se incluyó por su situación social. Todos concurrieron junto con sus madres; solo 6, con ambos padres; 12 lo hicieron, además, con otro familiar (abuela, tía); y 7, con los hermanos. Solo 5 familias participaron completas. Las madres presentaban bajo nivel de escolaridad: $53,12 \%$ había completado el primario (muchas analfabetas funcionales); $6,2 \%$ eran analfabetas, y solo el $28 \%$ había completado el secundario, sumado a que eran población joven. El 68,65\% habían hecho 5 o más controles de embarazo; el 6,25\% no se habían controlado y eran adolescentes. El 78,12\% vivía en pareja, pero inestables porque la mayoría de estas se habían formado al quedar embarazada la mujer y luego se habían separado.

De igual modo, los padres trabajaban temporariamente o vivían de pensiones o planes 
sociales, sin la posibilidad de tener una visión clara de futuro (Tabla 1).

\section{HERRAMIENTAS DE EVALUACIÓN}

1. TADI. Creado y validado en Chile, donde la población es bastante similar a la argentina. Instrumento muy rico, de bajo costo y puede ser aplicado por diferentes profesionales. El rango etario evaluable va desde los 3 meses y un día hasta los 6 años de edad. Evalúa las áreas motora, lenguaje, cognición y socioemocionalidad, en forma particular, y se realiza luego un promedio de las cuatro (puntaje T).

Estos resultados permiten ubicar el desempeño de los niños evaluados en distintas categorías del desarrollo y aprendizaje: avanzado, normal, riesgo y retraso. Cuando el puntaje total es normal con algún área particular de riesgo/retraso, se categoriza "normal con rezago". Estas categorías son descriptivas y representan una calificación cualitativa, no numérica. Los pacientes de los talleres

TABla 1. Características demográficas de los participantes. $N=32$. (Base de datos del Consultorio de Seguimiento de Prematuros del Hospital)

\section{SITUACIÓN MATERNA}

1) Edad

$\leq 18$ años: 7 pacientes

$>$ 18-29 años: 18 pacientes

$\geq 30-39$ años: 5 pacientes

$\geq 40$ años: 2 pacientes

2) Número de controles prenatales

- $\geq 5: 22$

$\bullet \leq 4: 8$

- $\mathrm{S} / \mathrm{C}: 2$

3) Nivel de estudio materno

- $3^{\circ}$ o univ.: 0

- $2^{\circ}$ completo: 9

- $2^{\circ}$ incompleto: 2

- $2^{\circ}$ en curso: 2

- $1^{\circ}$ completo: 17

- Analfabetas: 2

4) Estado civil

- Solteras: 7

- En pareja: 23

- Casadas: 2

\section{SITUACIÓN PATERNA}

Trabaja (inestable): 12

No trabaja: 2

En relación de dependencia/oficio: 13

No convive (se desconoce): 5 fueron evaluados en todas las áreas antes y después de la experiencia, cuyos resultados se informan en este trabajo, con posteriores evaluaciones de acuerdo con el programa de seguimiento.

2. Observación directa del comportamiento vincular. Compromiso de los padres, que acompañan el proceso de cambio, grado de participación en los talleres, vínculo con sus hijos en las actividades propuestas, cambio positivo en la demostración/expresión de los afectos para con sus hijos.

3. Cuestionario confeccionado por el equipo para el responsable del grupo familiar. Se entregó a los padres al finalizar la experiencia. Constaba de una serie de preguntas sobre los talleres: elección del taller más gustado, manera en que se dictó, su comprensión y aplicación en los hogares, la experiencia vivida, sugerencias y los cambios que habían notado en sus hijos y hogares a partir de la concurrencia. Para su mejor interpretación, la calificación era numérica (del 0 al 10) y mediante emoticones que la resignificaban (Anexo 1 en formato electrónico).

\section{DESARROLLO DE LA EXPERIENCIA: TALLERES}

Se realizaron 5 talleres, desde julio a diciembre de 2015. Considerándose la distancia de residencia y el tiempo de traslado, el horario fue de 9 a 12 h. Se utilizó un aula amplia, con luz natural y climatizada, que se acondicionaba según la temática del taller.

Al no contar con personal especializado para realizar los talleres, fueron ideados, implementados y monitoreados por el mismo equipo, basados en la experiencia personal, $\mathrm{y}$ adecuados a las herramientas con las que contaban los pacientes y su grupo familiar. ${ }^{16} \mathrm{Se}$ los invitaba al "taller de juegos" mediante un mensaje de texto enviado a sus celulares desde el Servicio de Referencia-Contrarreferencia del Hospital.

Se trabajó directamente con los padres, e indirectamente con los niños, y se utilizó un estilo de modelo experiencial ${ }^{17}$ multimodular que abarcaba diferentes valores como temas y se trató de abordar también diferentes temáticas planificadas pedagógicamente en forma espiralada. Fueron pensadas respondiendo a las deficiencias que más observaba el equipo, de manera tal de optimizar el desarrollo en todas sus áreas a través de la incorporación o 
fortalecimiento de esos valores y que fueron los siguientes (Anexo 2 en formato electrónico):

- Taller 1: Introducción a los talleres. El valor del ser persona. Derechos de los niños.

- Taller 2: Alimentación saludable (biológica y social). El valor del compartir y la comunidad.

- Taller 3: El valor de la familia. Roles y responsabilidades. Escolaridad y cultura del trabajo.

- Taller 4: El valor de la lectura y el relato.

- Taller 5: El valor de la Navidad independientemente del credo (renacer, unión, perdón, paz).

Cada taller fue guiado en forma alternada por algún Servicio. El personal del equipo se mantuvo sin cambios, lo que facilitó la identificación de las familias con ellos. Manteniendo el debido nivel académico y asistencial, adecuaron el lenguaje y el discurso a las necesidades, fortalezas internas y nivel de entendimiento de los padres, considerando que una gran mayoría eran analfabetos funcionales o analfabetos. ${ }^{18,19}$

Se comenzaba con una introducción al tema a modo de presentación, seguida de una actividad, a veces, personal, y se culminaba con una puesta en común grupal y comunitaria. Se buscaron herramientas fáciles de usar y conseguir. Entre los recursos, se utilizaron los videos "El cuento no es chiste", ${ }^{20}$ de Luis Landriscina, y "Contando desde los márgenes", de José Luis Gallego, ${ }^{21}$ quien coordina talleres para inventar y contar cuentos, lo que facilita el proceso de creación e imaginación de historias y promueve la instalación de espacios de escucha en contextos de vulnerabilidad y marginalidad. Relata la experiencia de un padre preso al narrarle un cuento a su hijo desde la cárcel. Al término de estos videos y su discusión, las familias lograron realizar su propia historia y armaron su propio libro de cuentos para sus hijos.

En general, se incluyeron trabajos manuales y la experiencia del role-play y obra de teatro, y se expusieron situaciones personales con consejería entre los familiares. La tarea integradora final era comunitaria. Siempre se llevaban como recuerdo material significativo realizado en cada evento y se comprometían a intentar aplicar lo vivido en el taller. ${ }^{22,23}$

\section{RESULTADOS}

Todos los padres observaron un cambio positivo en el comportamiento de sus hijos luego de los talleres. Reconocieron que ellos mismos no jugaban con sus hijos porque no habían tenido esa experiencia con sus padres. Así, descubrieron actividades desconocidas para ellos, el potencial personal y la capacidad de realizar tantas cosas por sí mismos en beneficio propio y de sus hijos.

En el cuestionario que llenaron, todos los padres calificaron los talleres con 9-10 (MUY BIEN), salvo 2 con 6-8: uno para "Implementación en la casa de lo aprendido" y otro para "¿Has notado cambios en la crianza de tus hijos?". En "observaciones", escribieron comentarios positivos (Tabla 2).

En el TADI, con respecto a la evaluación inicial, todos optimizaron el puntaje $\mathrm{T}$ dentro de la categoría "normal" (excepto el paciente con retraso), como así también el de las áreas socioemocionalidad y cognición, lo que equiparó a las otras áreas. Se reflejó también en una mejor atención y colaboración durante la evaluación. De los 3 con riesgo en lenguaje, resolvieron 2, y el tercero fue derivado a Fonoaudiología para

TABLA 2. Comentarios escritos por los pacientes en las evaluaciones sobre los talleres

- "La obra de teatro estuvo muy buena; me enseñó los hábitos en la casa para mejorar".

- "Me gustó todo, sobre todo, cómo mi hija se desenvolvió".

- “Que más madres traigan a sus hijos porque se aprende mucho".

- "Me gustaron. Aprendí cómo se preparaba la comida".

- "Me gustaría que me enseñen para que yo les enseñe a mis hijos".

- "Gracias por todo y marcar nuestras manos me gustó más".

- “Me gustó el tema de la alimentación y el derecho de los niños".

- "Me gustan todos los talleres que vienen haciendo. Me gustaría que haya un taller de títeres y quisiera dar gracias a (...) porque ayudó mucho a mi hijo".

- "Me gusta porque se aprenden muchas cosas que les sirvieron a ellos".

- "Me gustó lo que vi".

- "Sobre el estímulo sobre nuestros hijos, compartir lo hace más seguro".

- "No sabía que iba a poder contarle un cuento a mi hijo... Mi mamá no tenía tiempo y nosotros solo íbamos a la escuela y volvíamos a la casa y nunca nos contó... Es muy lindo y estoy emocionado y contento". 
tratamiento; actualmente, con rezago. No se evidenciaron diferencias según el número de participantes del grupo familiar o el tipo de riesgo socioambiental (Tabla 3).

Los niños continuaron siendo evaluados hasta la actualidad y, en su gran mayoría, están escolarizándose por propia iniciativa de sus familiares, con el deseo de mejorar cada vez más.
Los puntajes T continúan normales, con buena evolución, y son más sociables e interactivos. También se evidencia el cambio positivo de los padres sostenido en el tiempo.

En la atención de los pacientes, el abordaje holístico optimizó la dinámica y el encuentro entre los padres, que fue importante porque lograron reconocerse en el otro. Desde el

TABLA 3. Resultados del test de aprendizaje y desarrollo infantil (TADI) antes y después de la realización de los talleres

\begin{tabular}{|c|c|c|c|c|c|c|c|c|c|c|c|c|c|}
\hline \multirow[b]{2}{*}{ Paciente } & \multirow[b]{2}{*}{ EG } & \multicolumn{5}{|c|}{ TADI 1} & \multicolumn{7}{|c|}{ TADI 2} \\
\hline & & Eco. & $\mathbf{T}$ & $\mathrm{C}$ & $\mathbf{M}$ & $\mathbf{L}$ & SE & Eco. & $\mathbf{T}$ & $\mathrm{C}$ & $\mathbf{M}$ & $\mathbf{L}$ & SE \\
\hline 1 & 36 & $8 \mathrm{~m}, 3 \mathrm{~d}$ & 47 & 41 & 57 & 47 & 43 & $14 \mathrm{~m}, 16 \mathrm{~d}$ & 50,5 & 49 & 60 & 47 & 46 \\
\hline 2 & 36 & $18 \mathrm{~m}, 10 \mathrm{~d}$ & 42,5 & 41 & 51 & 40 & 38 & $24 \mathrm{~m}, 13 \mathrm{~d}$ & 52,8 & 51 & 62 & 52 & 46 \\
\hline 3 & 36 & $8 \mathrm{~m}, 3 \mathrm{~d}$ & 42,25 & 41 & 48 & 40 & 40 & $14 \mathrm{~m}, 16 \mathrm{~d}$ & 46 & 45 & 50 & 47 & 42 \\
\hline 4 & 34 & $10 \mathrm{~m}, 8 \mathrm{~d}$ & 34 & 35 & 28 & 35 & 38 & $16 \mathrm{~m}, 16 \mathrm{~d}$ & 35,5 & 37 & 39 & 32 & 34 \\
\hline 5 & 35 & $18 \mathrm{~m}, 12 \mathrm{~d}$ & 40,4 & 41 & 48 & 35 & 38 & $23 \mathrm{~m}, 0 \mathrm{~d}$ & 46,5 & 48 & 55 & 40 & 43 \\
\hline 6 & 33 & $16 \mathrm{~m}, 1 \mathrm{~d}$ & 47 & 45 & 55 & 47 & 41 & $22 \mathrm{~m}, 16 \mathrm{~d}$ & 51 & 48 & 55 & 52 & 49 \\
\hline 7 & 36 & $8 \mathrm{~m}, 0 \mathrm{~d}$ & 50,5 & 49 & 55 & 52 & 46 & $14 \mathrm{~m}, 21 \mathrm{~d}$ & 52,5 & 53 & 55 & 52 & 50 \\
\hline 8 & 36 & $13 \mathrm{~m}, 14 \mathrm{~d}$ & 50,5 & 49 & 55 & 52 & 46 & $19 \mathrm{~m}, 3 \mathrm{~d}$ & 52,8 & 55 & 55 & 52 & 49 \\
\hline 9 & 35 & $14 \mathrm{~m}, 0 \mathrm{~d}$ & 50,5 & 49 & 55 & 52 & 46 & $30 \mathrm{~m}$ & 52,5 & 51 & 58 & 52 & 49 \\
\hline 10 & 35 & $23 \mathrm{~m}, 0 \mathrm{~d}$ & 50 & 44 & 62 & 48 & 46 & $28 \mathrm{~m}, 18 \mathrm{~d}$ & 56 & 48 & 65 & 57 & 54 \\
\hline 11 & 35 & $9 \mathrm{~m}, 1 \mathrm{~d}$ & 44,75 & 42 & 50 & 46 & 41 & $15 \mathrm{~m}, 4 \mathrm{~d}$ & 48 & 49 & 50 & 47 & 46 \\
\hline 12 & 32 & $9 \mathrm{~m}, 2 \mathrm{~d}$ & 47,25 & 42 & 53 & 46 & 41 & $15 \mathrm{~m}, 5 \mathrm{~d}$ & 51,5 & 49 & 60 & 47 & 50 \\
\hline 13 & 36 & $23 \mathrm{~m}, 1 \mathrm{~d}$ & 40,5 & 41 & 48 & 35 & 38 & $29 \mathrm{~m}, 0 \mathrm{~d}$ & 42,5 & 40 & 48 & 39 & 43 \\
\hline 14 & 36 & $16 \mathrm{~m}, 9 \mathrm{~d}$ & 44 & 45 & 43 & 47 & 41 & $22 \mathrm{~m}, 10 \mathrm{~d}$ & 53 & 51 & 55 & 52 & 54 \\
\hline 15 & 36 & $21 \mathrm{~m}, 15 \mathrm{~d}$ & 40,5 & 38 & 51 & 35 & 38 & $27 \mathrm{~m}, 15 \mathrm{~d}$ & 44 & 40 & 54 & 41 & 41 \\
\hline 16 & 36 & $21 \mathrm{~m}, 0 \mathrm{~d}$ & 48,25 & 44 & 58 & 48 & 43 & $27 \mathrm{~m}, 18 \mathrm{~d}$ & 53,3 & 53 & 58 & 52 & 50 \\
\hline 17 & 36 & $21 \mathrm{~m}, 15 \mathrm{~d}$ & 43,5 & 38 & 58 & 40 & 38 & $28 \mathrm{~m}, 16 \mathrm{~d}$ & 45 & 40 & 58 & 41 & 41 \\
\hline 18 & 36 & $13 \mathrm{~m}, 9 \mathrm{~d}$ & 42,75 & 41 & 50 & 41 & 39 & $20 \mathrm{~m}, 0 \mathrm{~d}$ & 46,3 & 44 & 55 & 43 & 43 \\
\hline 19 & 34 & $8 \mathrm{~m}, 19 \mathrm{~d}$ & 45,25 & 41 & 53 & 47 & 40 & $14 \mathrm{~m}, 23 \mathrm{~d}$ & 48,3 & 45 & 55 & 47 & 46 \\
\hline 20 & 34 & $8 \mathrm{~m}, 20 \mathrm{~d}$ & 43,75 & 41 & 53 & 43 & 38 & $14 \mathrm{~m}, 21 \mathrm{~d}$ & 48,3 & 45 & 55 & 47 & 46 \\
\hline 21 & 36 & $15 \mathrm{~m}, 24 \mathrm{~d}$ & 44,75 & 41 & 50 & 47 & 41 & $22 \mathrm{~m}, 28 \mathrm{~d}$ & 48,5 & 48 & 51 & 48 & 47 \\
\hline 22 & 34 & $18 \mathrm{~m}, 11 \mathrm{~d}$ & 48,25 & 44 & 58 & 48 & 43 & $25 \mathrm{~m}, 13 \mathrm{~d}$ & 53,3 & 53 & 58 & 52 & 50 \\
\hline 23 & 36 & $23 \mathrm{~m}, 24 \mathrm{~d}$ & 46,25 & 41 & 58 & 48 & 38 & $30 \mathrm{~m}, 14 \mathrm{~d}$ & 48,8 & 45 & 60 & 48 & 42 \\
\hline 24 & 35 & $8 \mathrm{~m}, 2 \mathrm{~d}$ & 43 & 41 & 48 & 43 & 40 & $15 \mathrm{~m}, 9 \mathrm{~d}$ & 46,3 & 45 & 50 & 44 & 46 \\
\hline 25 & 36 & $9 \mathrm{~m}, 0 \mathrm{~d}$ & 40,25 & 41 & 48 & 34 & 38 & $16 \mathrm{~m}, 0 \mathrm{~d}$ & 43,5 & 45 & 50 & 37 & 42 \\
\hline 26 & 34 & $8 \mathrm{~m}, 12 \mathrm{~d}$ & 40,25 & 41 & 42 & 40 & 38 & $11 \mathrm{~m}, 22 \mathrm{~d}$ & 47 & 49 & 50 & 46 & 43 \\
\hline 27 & 34 & $8 \mathrm{~m}, 13 \mathrm{~d}$ & 40,75 & 41 & 42 & 40 & 40 & $11 \mathrm{~m}, 16 \mathrm{~d}$ & 48 & 49 & 50 & 47 & 46 \\
\hline 28 & 36 & $17 \mathrm{~m}, 20 \mathrm{~d}$ & 47,25 & 45 & 55 & 47 & 42 & $24 \mathrm{~m}, 18 \mathrm{~d}$ & 55 & 51 & 58 & 57 & 54 \\
\hline 29 & 36 & $12 \mathrm{~m}, 11 \mathrm{~d}$ & 47 & 45 & 55 & 47 & 41 & $19 \mathrm{~m}, 12 \mathrm{~d}$ & 51,5 & 51 & 58 & 48 & 49 \\
\hline 30 & 36 & $14 \mathrm{~m}, 6 \mathrm{~d}$ & 47,25 & 45 & 55 & 47 & 42 & $21 \mathrm{~m}, 1 \mathrm{~d}$ & 52,5 & 51 & 58 & 52 & 49 \\
\hline 31 & 35 & $19 \mathrm{~m}, 0 \mathrm{~d}$ & 46,25 & 44 & 55 & 43 & 43 & $23 \mathrm{~m}, 25 \mathrm{~d}$ & 51 & 51 & 58 & 48 & 47 \\
\hline 32 & 36 & $11 \mathrm{~m}, 15 \mathrm{~d}$ & 47 & 45 & 55 & 47 & 41 & $17 \mathrm{~m}, 12 \mathrm{~d}$ & 51,8 & 51 & 55 & 52 & 49 \\
\hline
\end{tabular}

Categorías interpretativas de los puntajes T

\begin{tabular}{lll} 
Puntaje T & Categoría & Observación \\
\hline 60 o más & Avanzado & \\
Entre 40 y 59 & Normal & Puntaje total normal con alguna dimensión con puntaje de riesgo o retraso: normal con rezago \\
Entre 30 y 39 & Riesgo & \\
29 o menos & Retraso & \\
\hline
\end{tabular}

Referencias:

Eco: edad corregida; EG: edad gestacional; m: meses (ej.: 9 m); d: días (ej.: 9 d); T: puntaje total; C: cognición; M: motricidad; L: lenguaje; SE: socioemocionalidad. 
aprendizaje, se generó un refuerzo positivo permanente y se logró la integración de los padres a la crianza y cuidado de sus hijos. A pesar de que tenían una menor adhesión a los controles en el Consultorio de Seguimiento, concurrieron al "taller de juegos", incluso en condiciones climáticas adversas, y retomaron los controles habituales. También solicitaron seguir con los talleres y sugirieron nuevos temas.

\section{COMENTARIO}

Por la alta demanda y una mejor atención y trabajo interdisciplinario, y también entre los mismos padres, se pensó en una prueba piloto y se idearon talleres de promoción del desarrollo infantil entre el Consultorio de Seguimiento y Servicio Social, con la colaboración de Nutrición. Significó un "desafío" ante la necesidad de intervenir, sobre todo, en lo humano, y se trató de lograr un cambio favorecedor. Además, con respecto a experiencias exitosas en otros países, esta era inédita en nuestro medio en cuanto a la edad de los pacientes (menores de 2 años), ya que no se contaba con un programa específico de este tipo. La problemática fue abordada desde el trabajo con los padres e indirectamente con los niños. Esto permitió conocer y reconocer la cultura interna de estas familias.

Como equipo de salud, se profundizó la relación interpersonal y profesional, y se enriquecieron y desarrollaron más las propias capacidades, desconocidas en muchos casos.

Independientemente del tipo de riesgo socioambiental, se corroboró la importancia de la intervención temprana en el período crítico del desarrollo. Mediante esta y la detección precoz, se favoreció el desarrollo global de estos niños, que, a lo largo de sus vidas, presentan mayores necesidades, más allá de lo meramente biológico, con una visión holística ${ }^{24,25}$ desde lo social, educacional y psicológico, necesidades que se amplían a su familia: la intervención sobre los padres puede favorecer la evolución cognitiva y socioemocional.

En Gestión de Salud, no es fácil mostrar y lograr la asimilación de la importancia del Consultorio de Seguimiento y de la intervención temprana. A pesar de darse, en un principio, a menor escala, con esta experiencia, se logró comprobar que, en nuestro medio, era igualmente posible, como en otros países de Europa, Brasil o Estados Unidos,${ }^{26}$ realizar un programa que favoreciera una adecuada crianza basada en el fortalecimiento afectivo-vincular de las familias, capaz de cambiar conductas poco adecuadas y desfavorables, en un futuro desenvolvimiento socioemocional adaptado al desarrollo de una sociedad más sana y equitativa, también desde lo económico. Esto impulsó el deseo de aplicarlo de manera sistemática y sostenida en el tiempo en el total de la población del Área Programática Este, lo que, actualmente, está en proceso de planificación.

La manera directa es trabajando en equipo, mediante la demostración de las potencialidades de los bebés desde que ingresan como tales. Se trata de que los padres descubran todo lo relacionado con la crianza de sus hijos de una manera diferente, nueva, y que salgan convencidos de que ellos pueden lograrlo a partir de la propia experiencia, incorporando pautas de puericultura, promoción de la escolaridad, incluso para ellos, y facilitando y promoviendo una cultura del trabajo, dando pistas para construir con sus propias herramientas nuevas formas de vida con calidez y calidad. En definitiva, la promoción humana tan ansiada en términos de equidad e igualdad para formar una sociedad unida y saludable como sustentable, tanto personal como social.

\section{REFERENCIAS}

1. Brazelton T. Justificación de la Intervención Temprana. Boston: Fundación Bernard van Leer, 1994.Pág.13.

2. Di Iorio S, Rodrigo M, Urrutia M. Desarrollo psicológico, nutrición y pobreza. Arch Argent Pediatr 1998;96(4):219-19.

3. Luby J, Belden A, Bolteron K, et al. The Effects of Poverty on Childhood Brain Development: The Mediating Effect of Caregiving and Stressful Life Events. JAMA Pediatr 2013;167(12):135-42.

4. Piaget J. La Psicología de la Inteligencia. Buenos Aires: Psique, 1973.

5. Piaget J. Seis estudios de psicología. Barcelona: Ariel, 1986.

6. Piaget J. El nacimiento de la inteligencia en el niño. Madrid: Aguilar, 1972.

7. Edwards M, Pardo M. Test de Aprendizaje y Desarrollo Infantil TADI. Centro de Estudios de Desarrollo y Estimulación Psicosocial CEDEP. Universidad de Chile, 2013. [Acceso: 12 de mayo de 2012]. Disponible en: https: / / tadi.cl/.

8. Schapira I, Toledo S, Roy E, et al. Los años formativos. Desarrollo e intervención oportuna en los primeros cinco años de vida. Buenos Aires: Fundación Neonatológica para el recién nacido y su familia, 2010.

9. Larguía M, Schapira I, Aspres N, et al. Guía para padres de prematuros. Buenos Aires: Fundación Neonatológica para el recién nacido y su familia, 2008.

10. Oiberman A, Mercado M. Nacer, jugar y pensar: guía para acompañar a los bebés desde su gestación hasta los tres años. Buenos Aires: Lugar, 2007.

11. Hermida MJ, Segretin MS, Lipina SJ, et al. Abordajes neurocognitivos en el estudio de la pobreza infantil: consideraciones conceptuales y metodológicas. Intern Jour Psych Psychol Therapy 2010;10(2):205-25.

12. Ministerio deSalud de la Nación-OPS. Indicadores básicos 
de Salud. Argentina 2011. Esperanza de vida Tucumán y Argentina. [Acceso: 12 de mayo de 2012]. Disponible en: http: / / publicaciones.ops.org.ar/publicaciones / indicadores/indicadores-nacion-2011.pdf.

13. Ministerio de Salud Pública de Tucumán. Documento de medición y monitoreo de indicadores de las metas de recursos humanos para la salud en la provincia de Tucumán. [Acceso: 17 de mayo de 2017]. Disponible en: http://www.msal.gob.ar/observatorio/images/stories/ documentos_red_federal/observatorios-provinciales / Tucuman/InformeTM-Tucuman-Dic2014.

14. UNICEF-Sociedad Argentina de Pediatría. Salud maternoinfantil-juvenil en Cifras. 2015. [Acceso: 12 de mayo de 2017]. Disponibleen:https: / / www.unicef.org/argentina/ spanish/SALUD_Sap-Unicef_2015_web.pdf.

15. Peralta MI, Muñoz C. La situación del trabajo infantil y su relación con las políticas públicas e intervención social en Argentina y Chile. Perspectivas 2006;17:75-122.

16. Vygotsky L. Pensamiento y lenguaje. Barcelona: Paidós Ibércia, 2010.

17. Dewey J. Experiencia y Educación. Madrid: Biblioteca Nueva, 2004.

18. Freire P. Pedagogía del oprimido. Buenos Aires: Siglo XXI, 1996.

19. Freire P. Pedagogía de la autonomía: saberes necesarios para la práctica educativa. $2^{\mathrm{da}}$ ed. Buenos Aires: Siglo XXI, 2008.

20. Landriscina L. El cuento no es chiste. [Acceso: 12 de mayo de 2017]. Disponible en: https://www.youtube.com/ watch?v=Ul_9ggGa6vA.

21. Gallego JL. Conferencia TEDx "Contando desde los márgenes". La Plata Argentina, 2013. [Acceso: 12 de mayo de 2017]. Disponible en: https://www.youtube.com/ watch? $v=$ L-eSrNZsZlE.

22. Sameroff AJ, Fiese BH. Transactional regulation: the developmental ecology of early intervention. In: Shonkoff JP, Meisels SJ (eds.). Handbook of early childhood intervention. 2nd ed. New York: Cambridge University Press, 2000. Págs.135-59.

23. Morasso MC, Duro E (Coord). Nutrición, Desarrollo y Alfabetización: una propuesta integradora en favor de la infancia. 2. ${ }^{\text {da }}$ ed. Buenos Aires: UNICEF, 2004. [Acceso: 12 de mayo de 2017]. Disponible en: https:/ / www.unicef. org/argentina/spanish/ar_insumos_NAD1.pdf.

24. BronfenbrennerU,CeciSJ.Nature-nurturereconceptualized: A bioecological model. Psychol Rev 1994;101(4):568-86.

25. Bronfenbrenner U. La Ecología del Desarrollo Humano. Barcelona: Paidós Ibérica, 1987.

26. Oates J. Apoyo a Los Padres. Aberystwyth: The Open University, 2010.

\section{"¿Dónde está la sabiduría que perdimos por el conocimiento?, ¿dónde está el conocimiento que perdimos por la información?"}




\section{ANEXO 1 \\ CUESTIONARIO ENTREGADO A LOS PADRES LUEGO DE LOS TALLERES}

TALLER DE CRIANZA

${ }^{*}$ Marca con una cruz la carita que te parece.

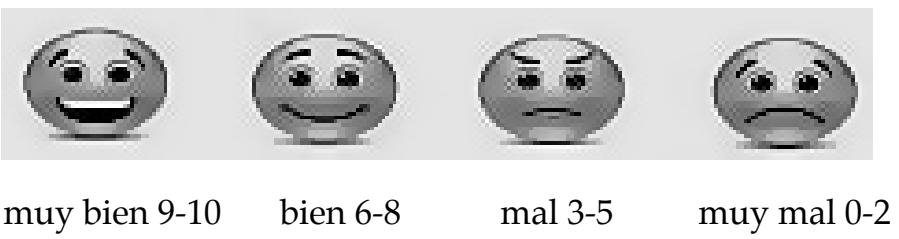

\section{CUESTIONARIO}

¿Cómo te sentiste en el taller?

¿Te gustaron los temas que se trataron?

¿Crees que el taller te ayudará a criar más tranquila a tu bebé?

¿Te gustó la manera en la que se lo dictó?

¿Te sirvió para aplicarlo en la casa?

¿Has visto cambios en la crianza de tus hijos?

¿Qué taller te gusto más?

1. Armado de sonajero. Derechos de los niños

2. Alimentación. Ensalada de frutas

3. Obra de teatro. La familia

4. Armado del libro. Lectura y relato

5. Navidad. Armado del arbolito

¿Alguna vez vivieron una experiencia de aprendizaje parecida?

(SÎ NO

¿Te gustaría tratar otros temas?

Sugerencias:.

Nombre y apellido:.. 


\section{ANEXO 2 \\ PLANIFICACIÓN DE LOS TALLERES}

TALLER 1: Introducción a los talleres. El valor de uno mismo (del ser persona)

- Objetivo:

1) Introducción a los talleres y conocimiento de los derechos de los niños.

2) Armado de un juguete casero (sonajero o similar).

- Propósito: que concienticen sobre el valor de ser uno mismo a través del descubrimiento de las propias capacidades artísticas e individuales.

- Actividades:

1) Charla inductiva a cargo de Servicio Social.

2) Intercambio de opiniones.

3) Armado de un sonajero.

- Materiales:

1) Presentación en PowerPoint.

2) Botella descartable de $500 \mathrm{ml}$, témperas, pinceles, brillantina, lentejuelas, fideos, agua, papeles de colores, goma eva, tijeras, pegamento, cinta adhesiva.

TALLER 2: Alimentación saludable. El valor del compartir y la comunidad

- Objetivo: concientizar sobre la importancia de la alimentación en el desarrollo biológico y también como un hecho social.

- Propósito: que mejoren la calidad de alimentación al compartir los padres sus propias experiencias y enriquezcan la "alimentación social".

- Actividades:

1) Cada familia dibuja un plato principal que usualmente cocinan.

2) Exposición de cada plato y los ingredientes usados con la observación y guía adecuada del enriquecimiento de las comidas.

3) Intercambio entre padres de sus experiencias y opiniones constructivas sobre alimentación saludable.

4) Charla sobre alimentación saludable.

5) Realización de una ensalada de frutas "comunitaria" con la participación de los padres y sus hijos.

6) Compartir la ensalada que hicieron entre todos.

- Materiales:

1) Cartulina con el dibujo impreso de un plato y cubiertos.

2) Revistas para recortar imágenes de frutas, verduras y condimentos saludables.

3) Láminas ilustrativas sobre alimentos saludables y su clasificación.

4) Frutas de estación, ensaladera grande, cuchillos, platos, servilletas, vasos, cucharas.

\section{TALLER 3: El valor de la familia (obra de teatro)}

- Objetivo: dar a conocer El valor de la familia.

- Propósito: que reconozcan a la familia como la primera comunidad básica, en la que se juegan roles, respeto, responsabilidad, compartir, crianza en general.

- Actividades:

1) Obra de teatro por parte de todos los Servicios. Tema: una familia disfuncional en el momento del mediodía. 
2) Trabajo en grupos sobre lo que observaron y qué sería lo más adecuado para un mejor funcionamiento entre los miembros de la familia.

3) Teatralización a cargo de los pacientes sobre los valores que enriquecen a la familia como tal.

- Materiales: Ideación del guion con conductas antisociales en el marco de una familia disfuncional a la hora del almuerzo. Escenografía y actores de reparto a cargo del personal de los 3 Servicios (Seguimiento, Servicio Social, Nutrición).

\section{TALLER 4: El valor de la lectura y el relato}

- Objetivo: presentar la lectura como el medio del desarrollo del lenguaje, la comunicación, la atención, la cognición, la ideación y el intercambio entre padres e hijos, y enriquecer su vinculación.

- Propósito:

1) Que los padres sean capaces de contar historias que puedan compartir con sus hijos y descubran tanto su propio potencial como la influencia positiva que brindan a sus hijos.

2) Descubrimiento del relato y su poder. Imaginación e ideación. Trabajo de la atención y colaboración.

- Actividades:

1) Charla y video de inducción de la importancia de la lectura y el cuento.

2) Relato de un cuento en forma oral junto con los padres guiado por el personal de

Seguimiento.

3) Introducción de Odontología en el cuento, aparición del "Sr. Muelitas" y finalización del cuento.

4) Armado de un libro de cuentos.

- Materiales:

1) Videos de TEDx (cuento en las cárceles) y Landriscina (una historia de la vida diaria).

2) Traje disfraz del Sr. Muelitas.

3) Cartón corrugado, cartulinas, témperas, pinceles, lápices y felpas, revistas, tijeras, plasticola, cinta adhesiva, lana de color, láminas impresas con dibujos para pintar.

TALLER 5: El valor de la Navidad independientemente del credo

- Objetivo: presentar la Navidad como la unión, el compartir, el perdón y la paz.

- Propósito: que incorporen la Navidad como un momento del año oportuno para recomenzar de la mejor manera posible, independientemente del credo, y no un mero hecho comercial o de calendario.

- Actividades:

1) Charla inductiva a cargo de los 3 Servicios.

2) Intercambio de opiniones y vivencias.

3) Armado de un arbolito de Navidad de mesa para cada hogar.

4) Armado final de un árbol de Navidad "comunitario" con las pelotitas en el que cada uno selle su mano (padres, hijos, personal).

5) Entrega de un juguete a cada participante.

6) Colocación del árbol de Navidad comunitario en el hall central del Hospital en ofrecimiento de paz para la comunidad toda. 